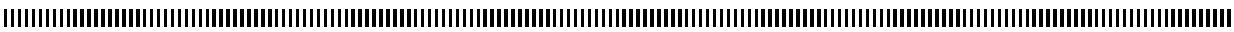

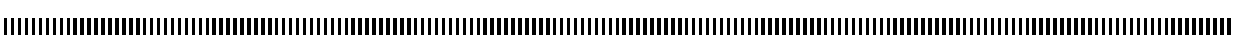

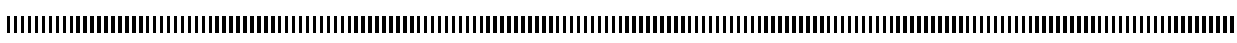

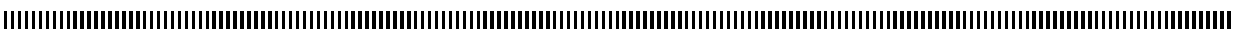

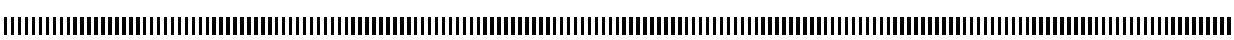

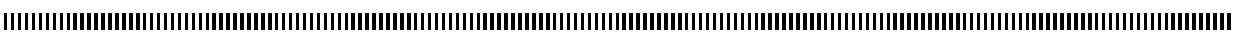

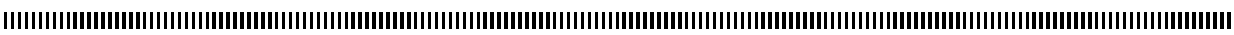

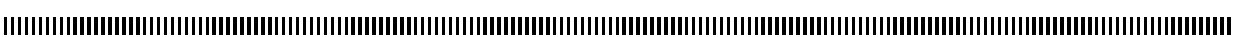
|||||||||||||||||||||||||||||||||||||||||||||||||||||||||||||||||||||||||||||||||||||||||||||||||||||||||||||||||||||||||||||||||||||||||||||||||||||||||||||||||||||||||||||||||||||||||||||||||||||||

\title{
Two numerical methods for Abel's integral equation with comparison
}

\author{
Abdallah Ali Badr \\ Department of Mathematics \\ University of Alexandria \\ Egypt \\ badrzoo@yahoo.com
}

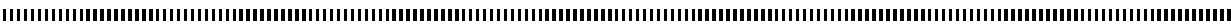

RÉSUMÉ. L'analogie entre équation intégrale de Abel et l'intégrale d'ordre fractionnaire d'une fonction donnee, $j^{\alpha} f(t)$, est discutée. Deux méthodes numériques différentes sont présentées et une formule d'approximation pour $j^{\alpha} f(t)$ est obtenue. La premiére approche considère le cas quand la fonction $f(t)$ est lisse et une formule quadrature est obtenue. Une formule modifièe est conçue dans le cas où la fonction a un ou plusieurs pôles simples. Dans la seconde approche, une procédure est présentèe pour affaiblir les singularités. Les deux approches peuvent être utilisées pour résoudre numériquement équation intégrale d'Abel. Quelques exemples numériques sont donnés pour illustrer nos résultats.

ABSTRACT. Analogy between Abel's integral equation and the integral of fractional order of a given function, $j^{\alpha} f(t)$, is discussed. Two different numerical methods are presented and an approximate formula for $j^{\alpha} f(t)$ is obtained. The first approach considers the case when the function, $f(t)$, is smooth and a quadrature formula is obtained. A modified formula is deduced in case the function has one or more simple pole. In the second approach, a procedure is presented to weaken the singularities. Both two approaches could be used to solve numerically Abel's integral equation. Some numerical examples are given to illustrate our results.

MOTS-CLÉS : equation intégral de Abel, intégrals fractional, polynômes de Jacobi, quadrature formule de Gauss-Jacobi.

KEYWORDS : Abel's integral equation, fractional integrals, Jacobi polynomials, Gauss-Jacobi quadrature formula.

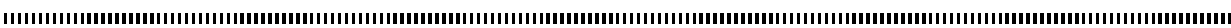

Received, May 18, 2010, 


\section{Introduction}

Many problems of mathematical physics and engineering lead to the Volterra integral equation ( VIE) of the first kind

$$
\int_{0}^{t} k(t, s) \phi(s) d s=f(t)
$$

where $f(t)$ and $k(t, s)$ are two given functions and $\phi(t)$ is an unknown function. The function $k(t, s)$ is called the kernel of this IE. As a special case of this IE is the linear Abel operator

$$
J_{\alpha} \phi(t)=\frac{1}{\Gamma(\alpha)} \int_{0}^{t}(t-s)^{\alpha-1} k(t, s) \phi(s) d s, \quad 0 \leq t \leq 1, \quad 0<\alpha<1 .
$$

In the case $k(t, s)=1$, the operator $J_{\alpha}$ is the classical Abel integral operator.

Theorem 1 : The solution of the classical Abel IE

$$
\frac{1}{\Gamma(\alpha)} \int_{a}^{x} \frac{g(t)}{(x-t)^{\alpha}} d t=f(x), \quad 0<\alpha \leq 1, \quad b>x>a
$$

is given by

$$
g(x)=\frac{1}{\Gamma(1-\alpha)} \frac{d}{d x} \int_{0}^{x}(x-u)^{\alpha-1} f(u) d u .
$$

Moreover, if $f(x)$ is absolutely continuous in $[a, b]$ then the solution is uniquely defined in $L_{1}[a, b]$. For treatises on the theory and applications of Abel IE we refer to [1].

Definition : Let $f(x) \in L_{1}([a, b])$, we define the fractional integral of order $\alpha, 1 \geq \alpha>$ 0 , of the function $f(x)$ over the interval $[a, b]$ as

$$
\begin{aligned}
J_{a^{+}}^{\alpha} f(x) & =\frac{1}{\Gamma(\alpha)} \int_{a}^{x} \frac{f(t)}{(x-t)^{1-\alpha}} d t, & & x>a, \\
J_{b^{-}}^{\alpha} f(x) & =\frac{1}{\Gamma(\alpha)} \int_{x}^{b} \frac{f(t)}{(t-x)^{1-\alpha}} d t, & & x<b .
\end{aligned}
$$

For completion, we define $J^{0}=I$ (identity operator), i.e. we mean $J^{0} f(x)=f(x)$. Furthermore, by $J_{a^{+}}^{\alpha} f(t)$ we mean the limit (if it exists) of $J_{c}^{\alpha}$ as $c \rightarrow a^{+}$.

This definition is according to Riemann-Liouville definition of fractional integral of arbitrary order $\alpha$.

For simplicity, we define the fractional integral by the equation

$$
J^{\alpha} f(x)=\frac{1}{\Gamma(\alpha)} \int_{a}^{x} \frac{f(t)}{(x-t)^{1-\alpha}} d t, \quad x>a, \quad 0<\alpha<1
$$

where we dropped $a^{+}$.

\section{Example :}

$$
J_{0^{+}}^{\alpha} t^{\gamma}=\frac{\Gamma(\gamma+1)}{\Gamma(\gamma+1+\alpha)} t^{\gamma+\alpha}, \quad 1>\alpha>0, \quad \gamma>-1, \quad t>0
$$


We see from this brief introduction that there is some analogy between Abel's IE and integral of a function of fractional order. In fact, the fractional integral of a function $f(x)$ of order $\alpha$ is just the Abel operator $J_{\alpha} f(t)$. Also, the operator

$$
D^{\alpha} f(x)=\frac{d}{d x} J^{1-\alpha} f(x)
$$

denotes the fractional derivative of $f(x)$ of order $\alpha$.

And so, any numerical treatment for the evaluation of such fractional integral will work nicely for solving Abel's IE.

The theory of fractional calculus, by Liouville in 1832, is a branch of mathematics which deals with the investigation of integrals and derivatives of arbitrary order, is considered as an old topic as the theory of Abel's IE, in 1826 by Abel. Recently, many scientists have remodeled some physical problems in terms of fractional integrals and fractional derivatives and a better result has been obtained, see a review article by Metzler and Klafter [4].

In this paper, we present two different approaches for approximating the classical Abel IE. In the first approach, we use Gaussian quadrature while in the second method, we use a well known method for removing singularities.

In section two, the first approach is presented and a quadrature formula is obtained for approximating the fractional integral $J^{\alpha} f(t)$ where the integrand is a smooth function and then a modified formula is obtained when the integrand has one or more simple singular point.

In section three, we describe a method to weaken the singular points of the integrand.

In section four, a numerical example is presented to illustrate our results and to compare between these two approaches.

\section{Method one : Quadrature formula for approximating $J^{\alpha} f(x)$}

\subsection{Case $1: f(x)$ is a smooth function}

Let $P_{n}^{(\lambda, \nu)}(x), n=0,1,2, \ldots$ denote the Jacobi polynomials of degree $n$ which form an orthonormal system over the interval $(-1,1)$ with respect to the weights function $(1-x)^{\lambda}(1+x)^{\nu}, \lambda, \nu>-1$;

$$
P_{n}^{(\lambda, \nu)}(1)=\frac{\Gamma(n+\lambda+1)}{n ! \Gamma(\lambda+1)} .
$$

The zeros of $P_{n}^{(\lambda, \nu)}(x)$, say $\left\{x_{k}\right\}_{1}^{n}$, are real, distinct and lie on $(-1,1)$. An n-point Gauss-Jacobi quadratic formula is given by, ( see [2], Eq. (7.1.2) and Eq.(7.3.3))

$\int_{-1}^{1}(1-x)^{\lambda}(1+x)^{\nu} \phi(x) d x \approx \sum_{k=1}^{n} A_{k} \phi\left(x_{k}\right)$

where the weights $A_{k}$ are given by :

$$
A_{k}=\int_{-1}^{1}(1-x)^{\lambda}(1+x)^{\nu} \frac{P_{n}^{(\lambda, \nu)}(x)}{\left(x-x_{k}\right) \cdot P_{n}^{(\lambda, \nu)^{\prime}}\left(x_{k}\right)} d x
$$




$$
=\frac{2^{\lambda+\nu+1} \Gamma(\lambda+n+1) \Gamma(\nu+n+1)}{n ! \Gamma(\lambda+\nu+n+1) \cdot\left[P_{n}^{(\lambda, \nu)^{\prime}}\left(x_{k}\right)\right]^{2}\left(1-x_{k}^{2}\right)} .
$$

Equation (2) is exact for all polynomials of degree up to $2 n-1$. This quadrature formula is valid if $\phi(x)$ possesses no singularities in $[-1,1]$, except for integrable singularities at the end points \pm 1 .

Setting $\nu=0$ and $\lambda=\alpha-1$ in equation (2), we have

$$
\int_{-1}^{1} \frac{\phi(x)}{(1-x)^{1-\alpha}} d x \approx G_{n}^{\alpha}(\phi)=\sum_{k=1}^{n} \frac{2^{\alpha}}{\left(1-x_{k}^{2}\right)\left[P_{n}^{(\alpha-1,0)^{\prime}}\left(x_{k}\right)\right]^{2}} \phi\left(x_{k}\right)
$$

and the error term is

$$
E_{n}^{\alpha}(\phi)=\int_{-1}^{1} \frac{\phi(x)}{(1-x)^{1-\alpha}} d x-G_{n}^{\alpha}(\phi) .
$$

If $\phi(x)$ has a continuous derivatives of order $n$ on the segment $[-1,1]$ then the reminder is

$$
E_{n}^{\alpha}(\phi)=\frac{\phi^{(2 n)}(\eta)}{(2 n) !} \frac{2^{2 n+\alpha}(n !)^{2}[\Gamma(n+\alpha)]^{2}}{(2 n+\alpha)[\Gamma(2 n+\alpha)]^{2}} .
$$

From definition of $A_{k}$, we deduce the following relation for Jacobi polynomials

$$
\int_{-1}^{1}(1-x)^{\alpha-1} \frac{P_{n}^{(\alpha-1,0)}(x)}{\left(x-x_{k}\right) \cdot P_{n}^{(\alpha-1,0)^{\prime}}\left(x_{k}\right)} d x=\frac{2^{\alpha}}{\left(1-x_{k}^{2}\right)\left[P_{n}^{(\alpha-1,0)^{\prime}}\left(x_{k}\right)\right]^{2}}
$$

On the other hand by transforming the segment $[a, x]$ into the segment $[-1,1]$, the integral $\int_{a}^{x} \frac{f(t)}{(x-t)^{1-\alpha}} d t$ can be written as

$$
\left(\frac{x-a}{2}\right)^{\alpha} \int_{-1}^{1} \frac{\phi(s) d s}{(1-s)^{1-\alpha}}
$$

where

$$
\phi(s)=f\left(\left(\frac{x-a}{2}\right) s+\left(\frac{x+a}{2}\right)\right) .
$$

This transformation allows us to rewrite the fractional integral $J^{\alpha} f(x)$ as

$$
J^{\alpha} f(x)=\frac{1}{\Gamma(\alpha)} \cdot\left(\frac{x-a}{2}\right)^{\alpha} \int_{-1}^{1} \frac{\phi(s)}{(1-s)^{1-\alpha}} d s .
$$

Using the Gauss-Jacobi quadrature (3), we obtain our first result

Theorem 2 : If $f(x) \in C^{n}[a, b]$, then

$$
J^{\alpha} f(x)=G_{n}^{\alpha}(f)+E_{n}^{\alpha}(f)
$$

where

$$
G_{n}^{\alpha}(f)=\frac{(x-a)^{\alpha}}{\Gamma(\alpha)} \sum_{k=1}^{n} \frac{f\left(\left(\frac{x-a}{2}\right) x_{k}+\left(\frac{x+a}{2}\right)\right)}{\left(1-x_{k}^{2}\right)\left[P_{n}^{(\alpha-1,0)^{\prime}}\left(x_{k}\right)\right]^{2}}
$$

and the error term is given by

$$
E_{n}^{\alpha}(f)=\frac{(x-a)^{\alpha}}{\Gamma(\alpha)} \frac{f^{(2 n)}\left(\left(\frac{x-a}{2}\right) \eta+\left(\frac{x+a}{2}\right)\right)}{(2 n) !} \frac{2^{2 n}(n !)^{2}[\Gamma(n+\alpha)]^{2}}{(2 n+\alpha)[\Gamma(2 n+\alpha)]^{2}} .
$$




\subsubsection{The error $E_{n}^{\alpha}(f)$}

To prove that $E_{n}^{\alpha}(f) \rightarrow 0$ as $n \rightarrow \infty$, we refer to the following theorem :

Theorem 3 : (for the proof see [2], page 248) :

Let $[a, b]$ be a finite segment. If $f(x)$ is an analytic function which is holomorphic in a certain region, [a,b], containing the set $\beta,(a, b)$, in its interior then for any function $\sigma$ the interpolatory quadrature process defined by Eq.(5) converges.

\subsection{Case $2: f(x)$ has a finite number of singular points}

In this section, we consider an analytic function, $\phi(z) z=x+i y$, within a closed contour $\mathrm{C}$ containing the interval $[-1,1]$ in its interior, except at some finite number of simple poles.

Let $\left\{z_{k}\right\}_{k=1}^{m}$ be those poles with corresponding residues $\left\{r_{k}\right\}_{k=1}^{m}$. We also assume that no one of the poles of $\phi(z)$ coincides with the zeros of $P_{n}^{(\alpha, \nu)}(x)$, otherwise, we use different value of $n$.

Applying the Cauchy residue theorem to the contour integral $\frac{1}{2 \pi i} \int_{c} \frac{\phi(z)}{P_{n}^{(\alpha-1,0)}(z)(z-x)} d z$, we obtain

$$
\begin{gathered}
\phi(x)=\sum_{k=1}^{n} \frac{\phi\left(x_{k}\right) P_{n}^{(\alpha-1,0)}(x)}{\left[P_{n}^{(\alpha-1,0)}\left(x_{k}\right)\right]^{\prime} \cdot\left(x-x_{k}\right)}+\sum_{k=1}^{m} \frac{r_{k} \cdot P_{n}^{(\alpha-1,0)}(x)}{P_{n}^{(\alpha-1,0)}\left(z_{k}\right) \cdot\left(x-z_{k}\right)} \\
+\frac{P_{n}^{(\alpha-1,0)}(x)}{2 \pi i} \cdot \int_{C} \frac{\phi(z)}{P_{n}^{(\alpha-1,0)}(z)(z-x)} d z .
\end{gathered}
$$

Multiplying both sides of the last equation by $(1-x)^{\alpha-1}$, we have

$$
\begin{aligned}
& \frac{\phi(x)}{(1-x)^{(1-\alpha)}}=\sum_{k=1}^{n} \frac{\phi\left(x_{k}\right)}{\left[P_{n}^{(\alpha-1,0)}\left(x_{k}\right)\right]^{\prime}} \cdot \frac{P_{n}^{(\alpha-1,0)}(x)}{\left(x-x_{k}\right)(1-x)^{1-\alpha}} \\
& +\sum_{k=1}^{m} \frac{r_{k}}{P_{n}^{(\alpha-1,0)}\left(z_{k}\right)} \cdot \frac{P_{n}^{(\alpha-1,0)}(x)}{\left(x-z_{k}\right)(1-x)^{1-\alpha}}+\frac{1}{2 \pi i} \cdot \frac{P_{n}^{(\alpha-1,0)}(x)}{(1-x)^{1-\alpha}} \cdot \int_{C} \frac{\phi(z)}{P_{n}^{(\alpha-1,0)}(z)(z-x)} d z .
\end{aligned}
$$

Integrating this equation over a path containing the interval $[-1,1]$ with a small semi circular indentation of radius $\epsilon$ above each of the pole $\left\{z_{k}\right\}_{k=1}^{m}$ and letting $\epsilon \rightarrow 0$ then using Eq.(3) and Eq.(4), we obtain

$$
\text { P.V. } \int_{-1}^{1} \frac{\phi(s) d s}{(1-s)^{1-\alpha}}=G_{n}^{\alpha}(\phi)+H_{n, m}^{\alpha}(\phi)+R_{n}^{\alpha}(\phi)
$$

where

$$
\begin{aligned}
& H_{n, m}^{\alpha}(\phi)=\sum_{k=1}^{m} \frac{r_{k}}{P_{n}^{(\alpha-1,0)}\left(z_{k}\right)} \text { P.V. } \int_{-1}^{1} \frac{P_{n}^{(\alpha-1,0)}(x)}{\left(x-z_{k}\right)(1-x)^{1-\alpha}} d x, \\
& R_{n}^{\alpha}(\phi)=\frac{1}{\pi i} \int_{C} \frac{\phi(z)}{P_{n}^{(\alpha-1,0)}(z)}\left\{\frac{1}{2} \int_{-1}^{1} \frac{P_{n}^{(\alpha-1,0)}(x) d x}{(z-x)(1-x)^{1-\alpha}} d z\right\} .
\end{aligned}
$$

Thus, as we have done in the previous section, the following theorem is proved:

Theorem 4 : If $f(x)$ is an analytic function in the interval $[a, b]$ except at one or more simple poles in the interval $(a, b)$, say $\left\{z_{k}\right\}_{k=1}^{m}$, then

$$
J^{\alpha} f(x) \approx G_{n, m}^{\alpha}(f), \quad 0<\alpha<1
$$


where

$$
G_{n, m}^{\alpha}(f)=G_{n}^{\alpha}(f)+H_{n, m}^{\alpha}(f),
$$

$G_{n}^{\alpha}(f)$ is given by Eq.(2), and $H_{n, m}^{\alpha}(f)$ is given by

$$
H_{n, m}^{\alpha}(f)=\frac{2}{\Gamma(\alpha)}\left(\frac{x-a}{2}\right)^{\alpha}\left\{\sum_{k=1}^{m} \frac{r_{k} q_{n}^{(\alpha-1,0)}\left(z_{k}\right)}{P_{n}^{(\alpha-1,0)}\left(z_{k}\right)}\right\} .
$$

where, see [7]

$$
q_{n}^{(\alpha-1,0)}(z)=\frac{1}{2} P . V . \int_{-1}^{1} \frac{(1-x)^{\alpha-1} P_{n}^{(\alpha-1,0)}(x)}{(x-z)} d x .
$$

The error term in this rule is

$$
E_{n}^{\alpha}(f)=\frac{1}{\pi i} \int_{C} \frac{q_{n}^{(\alpha-1,0)}(z)}{P_{n}^{(\alpha-1,0)}(z)} f(z)(z) d z .
$$

It is easy to show that the error $E_{n}^{\alpha}(f) \rightarrow 0$ as $n \rightarrow \infty$.

\section{Method two : Weakening the singularities of the integrand}

In this section we follow a well known approach to weaken the singularities of the integrand of Eq.(1).

\subsection{Case $1: f(x)$ is a smooth function}

Assume that $f(x)$ has derivatives up to certain order $m$ in the interval $[a, b]$ and that $f(x)$ does not vanish. Hence, the integral

$$
J^{\alpha} f(x)=\frac{1}{\Gamma(\alpha)} \int_{a}^{x} \frac{f(t)}{(x-t)^{1-\alpha}} d t, \quad x>a, \quad 0<\alpha<1
$$

is singular at the point $t=x$. We weaken the singularity of this integral by splitting its integrand into two parts as follows :

$$
F(t)=(x-t)^{\alpha-1} f(t)=F_{1}(t)+F_{2}(t)
$$

where,

$$
\begin{gathered}
F_{1}(t)=(x-t)^{\alpha-1}\left[\sum_{i=0}^{k-1} \frac{(-1)^{i} f^{(i)}(x)}{i !}(x-t)^{i}\right], \quad(k<m), \\
F_{2}(t)=(x-t)^{\alpha-1}\left[f(t)-\sum_{i=0}^{k-1} \frac{(-1)^{i} f^{(i)}(x)}{i !}(x-t)^{i}\right] .
\end{gathered}
$$

Thus

$$
J^{\alpha} f(x)=I_{1}+I_{2}
$$

where

$$
I_{1}=\frac{1}{\Gamma(\alpha)} \int_{a}^{x} F_{1}(t) d t, \quad I_{2}=\frac{1}{\Gamma(\alpha)} \int_{a}^{x} F_{2}(t) d t
$$


The integral $I_{1}$ can be calculated exactly by elementary calculations. In fact,

$$
I_{1}=\sum_{i=0}^{k-1} \frac{(-1)^{i+1} f^{(i)}(x)}{i ! \Gamma(\alpha+i)}(x-a)^{i+\alpha}
$$

Since the function $F_{2}(x)$ is differentiable $k$ more times than $f(x)$. Therefore,

$$
I_{2}=\int_{a}^{x}(x-t)^{\alpha-1}\left[f(t)-\sum_{i=0}^{k-1} \frac{(-1)^{i} f^{(i)}(x)}{i !}(x-t)^{i}\right] d t
$$

can be evaluated with a greater accuracy than $J^{\alpha} f(t)$ by a quadrature formula (Simpson rule for example).

\subsection{Case $2: \mathbf{f}(\mathbf{x})$ has a finite number of singular points in $[a, b]$}

Assume that $f(x)$ has singularities at several points in the interval $[a, b]$, say $x_{2}, x_{3}, \ldots, x_{n}$ with multiplicity $\beta_{i}, i=2,3, \ldots, n$. A similar procedure can be carried out in this case.

In fact, let

$$
F(x)=\phi(x)\left(x-x_{1}\right)^{\beta_{1}} \cdot\left(x-x_{2}\right)^{\beta_{2}} \ldots . .\left(x-x_{n}\right)^{\beta_{n}}
$$

where $x_{1}=x, \beta_{1}=\alpha-1$ and $\phi(x)$ is a smooth function. Define

$$
F_{j}(t)=\frac{F(x)}{\left(t-x_{j}\right)^{\beta_{j}}}, j=1,2, \ldots, n .
$$

Expanding the function $F_{j}(t)$ in a Taylor power series at $x_{j}$ gives

$$
F_{j}(t)=\sum_{i=0}^{k_{j}-1} \frac{F_{j}^{(i)}\left(x_{j}\right)}{i !}\left(t-x_{j}\right)^{i}, \quad j=1,2, \ldots, n .
$$

As before, we can split the integral into two parts

$$
J^{\alpha} f(x)=\frac{1}{\Gamma(\alpha)}\left(I_{1}+I_{2}\right)
$$

where

$$
\begin{gathered}
I_{1}=\int_{a}^{x} \sum_{i=1}^{n} F_{i}(t) d t \\
I_{2}=\int_{a}^{x}\left[F(t)-\sum_{i=1}^{n} F_{i}(t)\right] d t .
\end{gathered}
$$

The first integral is exactly evaluated. The integrand of the second integral has higher order derivatives than $f(t)$ and so a quadrature formula may be applied. 


\section{Example}

$$
\text { If } f(t)=\frac{1}{t-2}, x<2 \text {, then } J^{\alpha} f(x)=\frac{-x^{d}}{2 \Gamma(1+\alpha)}{ }_{2} F_{1}\left(1,1,1+d ; \frac{x}{2}\right) .
$$

In Table (1), we list the results of evaluating $J^{\alpha} f(x), \alpha=0.75$ for different values of $x$. Column one gives the exact value. Column three and four gives the errors resultant from applying method one for $N=4,18$ and these errors are denoted by $E_{4}^{1}, E_{18}^{1}$ respectively where $N$ is the the degree of Jacobi polynomial. In the last two columns, the errors resultant from applying method two for $k=2,3$ and they are denoted by $E_{2}^{2}, E_{3}^{2}$ respectively where $k$ is the number of terms in Taylor expansion of the integrand. Table (2) is the same as Table (1) except we take $\alpha=0.5$.

\section{Conclusion}

From our computations of the numerical results of this examples and others, we can conclude the following :

1- Both the presented methods could be used to evaluate integral with fractional order. 2- Method one has many difficulties : finding the values of the function at some points, evaluating its residues and evaluating the integral $q_{n}^{(\alpha-1,0)}(z)$. Moreover, as $N$ gets larger, the roots of the orthogonal polynomial get very close to each other and with computer rounding they become equal and hence the result gets worst for large $N$. In fact, this situation is solvable either by the appropriate choice of $N$ or the formula Eq.(??) has to be modified using Hermit interpolation formula instead. Yet, method one has the advantages of being sum of $N$ terms and so a rough estimate could be obtained easily.

3- Method two splits the fractional integral into two parts, the first one can be evaluated exactly and the second one is easily calculated using any known method of numerical integration since the integrand will be smoother than the original $f(x)$ and so the obtained formula is left to the user. Of course the accuracy of this method will depend on the user choice. 
Table (1) : $\alpha=0.75$

\begin{tabular}{|c||c||c|c||c|c|}
\hline $\mathrm{X}$ & exact value & $E_{4}^{1}$ & $E_{18}^{1}$ & $E_{2}^{2}$ & $E_{3}^{2}$ \\
\hline 0.5 & -0.38023108774222 & $.84986194 \mathrm{e}-5$ & $.55642852 \mathrm{e}-9$ & $.20 \mathrm{e}-19$ & $.30 \mathrm{e}-19$ \\
\hline 0.6 & -0.45280660307046 & $.97462973 \mathrm{e}-5$ & $.64086822 \mathrm{e}-9$ & $.20 \mathrm{e}-19$ & $.20 \mathrm{e}-19$ \\
\hline 0.7 & -0.52926296033339 & $.10952637 \mathrm{e}-4$ & $.72269666 \mathrm{e}-9$ & $.20 \mathrm{e}-19$ & $.20 \mathrm{e}-19$ \\
\hline 0.8 & -0.61086171417269 & $.12156905 \mathrm{e}-4$ & $.80247367 \mathrm{e}-9$ & $.40 \mathrm{e}-19$ & $.70 \mathrm{e}-19$ \\
\hline 0.9 & -0.69905686130528 & $.13473413 \mathrm{e}-4$ & $.88060123 \mathrm{e}-9$ & $.10 \mathrm{e}-19$ & $.70 \mathrm{e}-19$ \\
\hline 1.0 & -0.79564036203954 & $.15267997 \mathrm{e}-4$ & $.95738049 \mathrm{e}-9$ & $.40 \mathrm{e}-19$ & $.30 \mathrm{e}-19$ \\
\hline 1.1 & -0.90293824041614 & $.18708546 \mathrm{e}-4$ & $.103304455 \mathrm{e}-8$ & $.80 \mathrm{e}-19$ & .0 \\
\hline 1.2 & -1.0241118543254 & $.27492834 \mathrm{e}-4$ & $.110777854 \mathrm{e}-8$ & .0 & .0 \\
\hline 1.3 & -1.1636630846827 & $.53397159 \mathrm{e}-4$ & $.118173264 \mathrm{e}-8$ & $.10 \mathrm{e}-18$ & .0 \\
\hline 1.4 & -1.3283504049108 & $.13489144 \mathrm{e}-3$ & $.125503083 \mathrm{e}-8$ & .0 & .0 \\
\hline 1.5 & -1.5290022523089 & $.40408217 \mathrm{e}-3$ & $.132777692 \mathrm{e}-8$ & $.10 \mathrm{e}-18$ & $.20 \mathrm{e}-18$ \\
\hline 1.6 & -1.7845314222064 & $.13539972 \mathrm{e}-2$ & $.140006173 \mathrm{e}-8$ & $.20 \mathrm{e}-18$ & .0 \\
\hline 1.7 & -2.1323233670477 & $.50895380 \mathrm{e}-2$ & $.147256970 \mathrm{e}-8$ & $.10 \mathrm{e}-18$ & $.20 \mathrm{e}-18$ \\
\hline 1.8 & -2.6624729819275 & $.22898574 \mathrm{e}-2$ & $.180913911 \mathrm{e}-8$ & .0 & $.90 \mathrm{e}-17$ \\
\hline 1.9 & -3.6953991620052 & .15017889485 & $.490172941 \mathrm{e}-6$ & $.15 \mathrm{e}-17$ & $.5 \mathrm{e}-17$ \\
\hline \hline
\end{tabular}

Table (2) : $\alpha=0.5$

\begin{tabular}{|c|c|c|c||c|c|}
\hline $\mathrm{X}$ & exact value & $E_{6}^{1}$ & $E_{12}^{1}$ & $E_{4}^{2}$ & $E_{5}^{2}$ \\
\hline 0.5 & -0.48240083637052040 & $.62115253 \mathrm{e}-7$ & $.874090 \mathrm{e}-14$ & $.20 \mathrm{e}-19$ & $.20 \mathrm{e}-19$ \\
\hline 0.6 & -0.55277602012174696 & $.68044050 \mathrm{e}-7$ & $.940872 \mathrm{e}-14$ & $.10 \mathrm{e}-19$ & $.10 \mathrm{e}-19$ \\
\hline 0.7 & -0.62650228039198733 & $.73498070 \mathrm{e}-7$ & $.997543 \mathrm{e}-14$ & $.60 \mathrm{e}-19$ & .0 \\
\hline 0.8 & -0.70530496999699923 & $.78590277 \mathrm{e}-7$ & $.145624 \mathrm{e}-13$ & .0 & .0 \\
\hline 0.9 & -0.79110079140524638 & $.83482152 \mathrm{e}-7$ & $.135621 \mathrm{e}-13$ & $.50 \mathrm{e}-19$ & $.50 \mathrm{e}-19$ \\
\hline 1.0 & -0.88622692545041249 & $.88786282 \mathrm{e}-7$ & $.108610 \mathrm{e}-13$ & .0 & $.60 \mathrm{e}-19$ \\
\hline 1.1 & -0.99373557331290618 & $.97728830 \mathrm{e}-7$ & $.114874 \mathrm{e}-13$ & .0 & .0 \\
\hline 1.2 & -1.1178450040268358 & $.12773757 \mathrm{e}-6$ & $.122170 \mathrm{e}-13$ & $.10 \mathrm{e}-18$ & $.10 \mathrm{e}-18$ \\
\hline 1.3 & -1.2647088583999714 & $.27291811 \mathrm{e}-6$ & $.259846 \mathrm{e}-12$ & .0 & .0 \\
\hline 1.4 & -1.4438487639150942 & $.10527540 \mathrm{e}-5$ & $.381792 \mathrm{e}-12$ & .0 & $.10 \mathrm{e}-18$ \\
\hline 1.5 & -1.6710855155927290 & $.54998706 \mathrm{e}-5$ & $.320808 \mathrm{e}-09$ & $.40 \mathrm{e}-18$ & $.2 \mathrm{e}-18$ \\
\hline 1.6 & -1.9752907125248785 & $.33224852 \mathrm{e}-4$ & $.129948 \mathrm{e}-07$ & $.10 \mathrm{e}-18$ & $.520 \mathrm{e}-18$ \\
\hline 1.7 & -2.4167307171846061 & $.23516697 \mathrm{e}-3$ & $.849982 \mathrm{e}-06$ & $.42 \mathrm{e}-17$ & $.15 \mathrm{e}-17$ \\
\hline 1.8 & -3.1514963467007607 & $.21928576 \mathrm{e}-2$ & $.849982 \mathrm{e}-06$ & $.22 \mathrm{e}-16$ & $.344 \mathrm{e}-16$ \\
\hline 1.9 & -4.7993070810190729 & $.37731535 \mathrm{e}-1$ & $.161578 \mathrm{e}-03$ & $.27 \mathrm{e}-15$ & $.13989 \mathrm{e}-15$ \\
\hline
\end{tabular}

\section{Bibliographie}

[1] H.Brumner , P.J. VAn DeR Houwen« The Numerical Solution of Volterra Equations, North-Holland » 1986.

[2] V.I.KRYLOV Approximate Calculation of Integrals » The Macmillan Company, New York : translated by A.Stroud, 1962. 
10 ARIMA Journal - Volume $15-2012$

[3] F.MAinARd « Fractional Calculus : Some Basic Problem In Continum And Statistical Mechanics, In Fractals And Fractional Calculus In Continuum Mechanics » A. Carpinteri and F.Mainardi eds., Wien, Springer, 291-348, 1997.

[4] R.MetZleR, J.KLAFTER« The resturant at the end of the random walk : recent developments in the description of anomalous transport by fractional dynamics » J.phys. A 37, 161-208, 2004.

[5] L.Nishimoto « Tables of fractional differitegrations of elemantary functions »J., coll. Engng. Nihon Univ., B-25,41-46, 1984.

[6] H.SUgIURA, T. HASEGAWA « Quadrature rule for Abel"s equation : uniformly approximating fractional dervaives » BIT 46, 195-202, 2006.

[7] G. SzEGÖ« Orthogonal Polynomials, Amr. Math. Soc., Colloquim Publications, Vol. XXIII, Providence, R.I., (third edition) 1967.

[8] J.WALDVOGEL « Fast construction of the Fejér and Clenshaw - Curtis quadrature rule, BIT 43, 1-18, 2004. 